\title{
A Fluorescence System Composed of Nitrogen- doped Graphene Quantum Dots and Gold Nanoparticles Coated With Phenylalanine for Selective and Sensitive Quantification of Piroxicam in Biological Samples
}

Maryam Moallemi Bahmani

Yazd University

Ali Mohammad Haji Shabani

Yazd University

Shayessteh Dadfarnia ( $\nabla$ sdadfarnia@yazd.ac.ir)

Yazd University https://orcid.org/0000-0002-6191-0775

Roya Afsharipour

Yazd University

\section{Research Article}

Keywords: Piroxicam, Fluorimetric method, Nitrogen graphene quantum dots, Gold nanoparticles coated with phenylalanine

Posted Date: January 5th, 2022

DOI: https://doi.org/10.21203/rs.3.rs-1209906/v1

License: (c) (1) This work is licensed under a Creative Commons Attribution 4.0 International License. Read Full License 


\section{Abstract}

In this study, a sensitive fluorimetric method is proposed for the determination of piroxicam using nitrogen graphene quantum dots (N-GQDs) and gold nanoparticles coated with phenylalanine. The fluorescence emission of N-GQDs at $440 \mathrm{~nm}$ decreases with the increase of gold nanoparticles coated with phenylalanine. However, the addition of piroxicam causes the release of gold nanoparticles from the surface of quantum dots followed by the retrieval of the fluorescence emission of N-GQDs. Under the optimum conditions, the calibration graph was linear in the concentration range of $2.0-35.0 \mathrm{nmol} \mathrm{L^{-1 }}$ for piroxicam with a limit of detection of $0.11 \mathrm{nmol} \mathrm{L}^{-1}$. The developed method was successfully applied for the determination of piroxicam in urine and serum samples.

\section{Introduction}

Piroxicam (PX) is a non-steroidal anti-inflammatory drug (NSAID) that plays an important role in reducing pain in a variety of arthritis and other post-operative conditions [1]. Although, this drug is vastly employed in human and veterinary medicine, unfortunately, some common side effects including headache, gastrointestinal disorders, dizziness, palpitations, skin rashes, and tinnitus have been observed in the patients consuming this drug [2,3]. The increased request for its production increased its release as an important biological pollutant into the effluent of pharmaceutical industries. Thus, the design of a sensitive and selective method for quantification of the low amount of this compound in environmental samples as well as human blood and urine is necessary. Several analytical methods include spectrophotometry [4], liquid chromatography [5], spectrofluorometry [6], and capillary electrophoresis [7] were applied for the determination of piroxicam. Among the mentioned method, fluorescence techniques with the unique properties of high sensitivity, simple operation, and stability have attracted researchers in analytical and biological fields [8,9].

Fluorescence resonance energy transfer (FRET), introduced as a technology in fluorescence methods in which energy can be transferred from fluorophore as a donor to an acceptor via dipole-dipole coupling process $[10,11]$. This technique has been successfully utilized for the detection of different biological molecules [12-14] and ions [15,16], but there is no report on the use of this method for the determination of piroxicam. In the design of a FRET system, the selection of proper donor-acceptor pairs is important for the achievement of good efficiency.

Graphene quantum dots (GQDs) as a new kind of zero-dimensional carbon nanomaterial with oxygencontaining functional groups and sizes smaller than $100 \mathrm{~nm}$, has been employed in different fields including catalysis, biosensors, biological labeling, and bioimaging [17-22]. GQDs have distinct advantages of excellent biocompatibility, good water solubility, low toxicity, strong photoluminescence, and large surface area. Thus, in the design of fluorescent nanoprobes, GQDs are a promising alternative to the conventional quantum dots of heavy metals and carbon dots $[23,24]$. The numerous oxygencontaining hydroxyl and carboxyl groups, give the GQDs good capability of modification with functional groups making them favorable as electron acceptors and electron donors. 
Gold nanoparticles (Au NPs) with the great advantages of the excellent surface plasma resonance, obvious color change, and high extinction coefficient have been used in colorimetric and fluorimetric sensors [22,25]. The fabrication of fluorescence sensors based on gold nanoparticles and graphene quantum dots provides a sensitive and selective nanoprobe for the quantification of biomolecules and drugs $[22,25]$. The operation of these fluorescence sensors is based on the capability of Au NPs in quenching the fluorescence intensity of graphene quantum dots through the FRET mechanism [26].

Herein, blue emission nitrogen-doped graphene quantum dots were chosen as a donor, and gold nanoparticles coated with phenylalanine as an acceptor in the design of a fluorescent nanoprobe for the sensitive and selective FRET determination of piroxicam. The fluorescence intensity of N-GQDs was significantly quenched (off-status) in the presence of Au NPs coated with phenylalanine due to the fluorescence resonance energy transfer (FRET). However, the addition of the piroxicam resulted in the hydrogen bond formation between the functional groups of gold nanoparticles and drug followed by the release of Au NPs from the surface of quantum dots, and retrieval of fluorescent intensity of N-GQDs.

\section{Experimental}

\section{Materials and reagents}

Phenylalanine and Au (III) chloride trihydrate were supplied from Merck Company (Darmstadt, Germany, http://www.merckmillipore.com). Piroxicam was prepared from Sigma-Aldrich (Buenos Aires, Argentina). All materials were applied with the highest purity. A $50.0 \mu \mathrm{g} \mathrm{mL}^{-1}$ stock solution of piroxicam was prepared by diluting $2.5 \mathrm{mg}$ of piroxicam to $50 \mathrm{~mL}$ in a volumetric flask with double distilled water and stored in the refrigerator. Solution of phenylalanine $\left(25.0 \mathrm{mmol} \mathrm{L}^{-1}\right)$ was prepared by dissolving proper amounts of it in double-distilled water. Tetrachloroauric acid $\left(25.0 \mathrm{mmol} \mathrm{L}^{-1}\right)$ solution for synthesis of gold nanoparticles was also prepared and stored in the refrigerator.

\section{Apparatus}

A model FL-Ar fluorescence array spectrophotometer (Teifsanje Tajhiz Pishraftheh Co. Ltd., Iran, http://en.teifsanje.ir) was utilized for monitoring the fluorescence intensity. A model SPECORD 250, UVVis spectrophotometer (Analytik Jena AG, Germany, https://www.analytik-jena.com) was employed for recording the UV-Vis absorption spectra. The $\mathrm{pH}$ was adjusted using an inoLab $\mathrm{pH}$ meter (model 7110, Germany, https://www.xylemanalytics.com). A Bruker Vector 22 FT-IR spectrometer (Bruker Equinox 55, Germany, https://www.bruker.com) was used for recording the Fourier transform infrared (FT-IR) spectra. Transmission electron microscopy (TEM) images were achieved by a Zeiss-EM10C electron microscope with an accelerating voltage of $100 \mathrm{kV}$ (Zeiss, Germany, https://www. zeiss.com).

\section{Preparation of N-GQDs}

N-GQDs were prepared by the reported study [26]. The citric acid $(1.26 \mathrm{~g})$ and urea $(1.08 \mathrm{~g})$ were added into a backer containing $30.0 \mathrm{~mL}$ of double-distilled water and stirred until a clear solution was obtained. Then, the solution was transferred into a Teflon-lined stainless autoclave and heated at $170^{\circ} \mathrm{C}$ for 5 
hours. The product was centrifuged for 10.0 minutes at $3500 \mathrm{rpm}$ for removing the larger particles and stored in the refrigerator.

\section{Gold nanoparticles coated with phenylalanine preparation}

For the synthesis of the gold nanoparticles coated with phenylalanine, $300.0 \mu \mathrm{L}$ of phenylalanine solution as a reduction and modification reagent with the concentration of $25.0 \mathrm{mmol} \mathrm{L}^{-1}$ was added to a $100 \mathrm{~mL}$ beaker containing $15.0 \mathrm{~mL}$ of water, under stirring. The mixture was heated to the boiling point and while heated, $1.0 \mathrm{~mL}$ of tetrachloroauric acid solution $\left(1.0 \mathrm{mmol} \mathrm{L}^{-1}\right)$ was added to it. After a few minutes, the solution turned pinkish-red, and when the reaction has completed, the colloidal solution was rapidly cooled by placing the vessel and its content into an ice water bath. The prepared nanoparticles were stored in sealed dark containers in the refrigerator for further experiments.

\section{The procedure of determination of piroxicam}

For sensitive determination of piroxicam (PX), $500.0 \mu \mathrm{L}$ of N-GQDs, along with $200 \mu \mathrm{L}$ of PBS buffer (pH $=7.0$ ), $300.0 \mu \mathrm{L}$ of Au NPs coated with phenylalanine, and $500.0 \mu \mathrm{L}$ of the sample or standard solution of piroxicam were added into a quartz cell. After 10 minutes of incubation, the fluorescence intensity of $\mathrm{N}$ GQDs in the presence of piroxicam was measured at $440 \mathrm{~nm}(\mathrm{~F})$. The fluorescence intensity of a blank solution containing all reagents except the drug was also measured at $440 \mathrm{~nm}$ under the same experimental conditions $\left(\mathrm{F}_{0}\right)$. The $\left(\mathrm{F}-\mathrm{F}_{0}\right) / \mathrm{F}_{0}$ was proportion to the analyte concentration and was applied as the analytical signal for the quantification of the piroxicam.

\section{Sample preparation}

Serum and urine samples were provided by a healthy volunteer who in the past three months did not consume any drugs. The urine sample was prepared by centrifugation at $5000 \mathrm{rpm}$ for 15 minutes and passing the supernatant through a $0.45 \mu \mathrm{m}$ filter. Then, its piroxicam content was determined according to the given procedure.

For the preparation of the serum sample, $2.0 \mathrm{~mL}$ of acetonitrile was added to the $4.0 \mathrm{~mL}$ of the human serum sample, the mixture was centrifuged at $10000 \mathrm{rpm}$ for 10 minutes, the supernatant was transferred to a $25.0 \mathrm{~mL}$ volumetric flask and diluted to the mark with distilled water. Finally, its piroxicam content was measured by the developed procedure.

To prepare a sample of piroxicam capsules, the contents of 10 capsules of piroxicam ( $10 \mathrm{mg} / \mathrm{capsules})$ were mixed and ground well. Then, a precise amount of it, equivalent to one-tenth of the weight of one capsule $(0.1652 \mathrm{~g})$ was put into a beaker, some water was added to it, and after sonicating for 10 minutes, was passed through a $0.45 \mu \mathrm{m}$ Millipore filter. Then, the filtrate was diluted properly with double distilled water to get a solution with the drug concentration within the linear range of the calibration graph. The amount of piroxicam was determined according to the given procedure. 


\section{Results And Discussion}

\section{Characterization of N-GQDs}

The characterization of N-GQDs synthesized by the one-step hydrothermal method was done by FT-IR, UVVis spectra, $x$-ray diffraction (XRD), and transmission electron microscopy (TEM).

The FT-IR spectrum of N-GQDs is shown in Figure 1a. The broad absorption band appearing at the 3315 $\mathrm{cm}^{-1}$ corresponds to the $\mathrm{O}-\mathrm{H}$ and $\mathrm{N}-\mathrm{H}$ stretching vibration, respectively, and the band observed at the $1090 \mathrm{~cm}^{-1}$ corresponds to the $\mathrm{C}-0$ stretching vibrations. The bands that appeared at 2372 and 1636 , 1576 , and $1388 \mathrm{~cm}^{-1}$ are related to $\mathrm{C}-\mathrm{H}, \mathrm{NH}-\mathrm{CO}, \mathrm{C}-\mathrm{C}$, and $\mathrm{C}-\mathrm{N}$ stretching vibrations, respectively. Thus, the FT-IR spectrum of N-GQDs indicates its successful synthesis.

The absorption spectrum of the synthesized N-GQDs (Fig. 2b) exhibits a strong absorption band with a maximum at $235 \mathrm{~nm}$ related to the $\pi-\pi^{\star}$ transitions of aromatic $\mathrm{C}=\mathrm{C}$ bond $\left(\mathrm{sp}^{2}\right)$, and another strong absorption band with a maximum at $342 \mathrm{~nm}$ due to the $\mathrm{n}-\pi^{*}$ transition of $\mathrm{C}=0$ bond. The appearance of the absorption maximum in the blue region of the spectrum is an indication of the small size of the synthesized N-GQDS.

TEM image of synthesized N-GQDs (Fig. 1c), shows the formation of spherical particles with uniform distribution. According to Fig. 1c, the dimensions of N-GQDs are in the range of 3.3-5.3 nm with an average diameter of $4.3 \mathrm{~nm}$.

The XRD pattern of the N-GQDs is provided in Figure $1 \mathrm{~d}$. In this pattern, a broad diffraction peak (002) with a maximum at $2 \theta$ of about $24.3^{\circ}$ is observed which is related to the graphite structure and proves the formation of these quantum dots.

\section{The role of amino acids in the preparation of gold nanoparticles}

Alpha-amino acids are compounds that differ in the type of group in their alpha position. In this study, the amino acid phenylalanine was used as a coating and reducing agent to prepare Au NPs. Thus, in the preparation of gold nanoparticles, some amino acids act as a reducing agent and the excess amino acid binds to the surface of the gold nanoparticles (Fig. 2). The gold atoms acting as Lewis acid coordinate with the unbonded electron pair of the amino group of phenylalanine. Functionalization of Au NPs with amino acids resulted in the stabilization and dispersion of the nanoparticles in water. The presence of amino acids on the surface of Au NPs with functional groups of $\mathrm{O}-\mathrm{C}=\mathrm{O}$ and $\mathrm{N}-\mathrm{H}$ is useful for binding it to other molecules.

\section{UV-Vis spectrum of Au NPs coated with phenylalanine as a function of times}

One of the methods of recognition of Au NPs is the study of its UV-Vis spectrum. Gold nanoparticles have an absorption band in the range of 500 to $600 \mathrm{~nm}$ with a $\lambda_{\max }$ that depends on the size of the nanoparticles. To investigate the formation of gold nanoparticles, the absorption of the sample was 
recorded at different times interval from the start of synthesis (Fig. 1S). As it is observed, when the time is increased up to 20 minutes, the absorption intensity is increased and the $\lambda_{\max }$ is shifted toward the red wavelength. But, further increase in time has no noticeable effect on the $\lambda_{\max }$ or intensity of absorption, and the fluorescence intensity becomes constant at the wavelength of $552 \mathrm{~nm}$. For the explanation of this observation, the gold nanoparticles can be assumed as clusters made of gold atoms. Then, according to the bond theory, the greater the number of gold atoms connected, the smaller the distance between HUMO and LUMO in the clusters, and the less energy required for the electron transfer between these two levels, resulting in a shift in maximum absorption toward the longer wavelength. Thus, the $\mathrm{Au}$ NPs coated with phenylalanine have a $\lambda_{\max }$ of absorption at $522 \mathrm{~nm}$ with a synthesis time of 20 minutes.

\section{Characterization of Au NPs coated with phenylalanine}

To confirm the successful binding of phenylalanine on the surface of the Au NPs, FT-IR analysis was performed. A comparison of the spectra of phenylalanine, Au NPs, and Au NPs coated with phenylalanine (Fig. 2S) revealed that the spectrum of Au NPs coated with phenylalanine has an absorption band at $3354 \mathrm{~cm}^{-1}$ related to the $\mathrm{OH}$ group on the surface of Au NPs. Furthermore, the absorption band at 1646, 1544 , and $1211 \mathrm{~cm}^{-1}$ are related to the $\mathrm{C}=0$ group, the aromatic $\mathrm{C}=\mathrm{C}$ of the phenylalanine benzene ring, and the stretching vibration of $\mathrm{C}-\mathrm{O}$ and $\mathrm{C}-\mathrm{N}$ of the phenylalanine, respectively. The above results indicate the successful binding of phenylalanine to the surface of Au NPs.

The TEM image of gold nanoparticles coated with phenylalanine (Fig. 2S) shows that these nanoparticles are spherical with an average diameter of $37.8 \pm 0.5 \mathrm{~nm}$.

\section{Optical N-GQDs sensor for piroxicam determination}

To evaluate how the system works and present a suitable mechanism, various experiments were done. In the first experiment, $500.0 \mu \mathrm{L}$ of N-GQDs, $300.0 \mu \mathrm{L}$ of phenylalanine $\left(25.0 \mathrm{mmol} \mathrm{L}^{-1}\right), 200.0 \mu \mathrm{L}$ of phosphate buffer $(\mathrm{pH}=7.0)$, and $500 \mu \mathrm{L}$ of piroxicam solution with the concentration of $11.0 \mathrm{nmol} \mathrm{L}^{-1}$ was transferred into a quartz cell and after 10.0 minutes its fluorescence was recorded. The blank solution was prepared with the same ingredient except for the drug and its fluorescence under the same conditions was recorded. As shown in Fig. 3a, the addition of phenylalanine causes a decrease in the fluorescence intensity of N-GQDs at $440 \mathrm{~nm}$ due to the formation of hydrogen bonds between the amino group of the amino acid and the acidic and amide groups of N-GQDs. The addition of piroxicam to this system resulted in a further decrease in the fluorescence intensity due to the hydrogen bond formation between the drug and phenylalanine.

The second experiment was done as the previous one but instead of phenylalanine solution, $300.0 \mu \mathrm{L}$ distilled water was used. The results (Fig. 3b) revealed that upon addition of the drug, the fluorescence intensity of N-GQDs slightly is reduced due to the hydrogen bond formation between the drug and hydroxyl and amide groups on the N-GQDs surface. 
The third experiment was performed as the first one but $300.0 \mu \mathrm{L}$ of Au NPs was used instead of phenylalanine solution. The result of this investigation (Fig. 3c) shows that the addition of Au NPs resulted in a decrease in the fluorescence intensity of N-GQDs due to the resonance energy transfer mechanism. However, the addition of piroxicam to this mixture has no significant effect on the fluorescence intensity, indicating that piroxicam can not directly bond to the Au NPs.

The fourth experiment was done as the first one but the phenylalanine solution was replaced with a 300.0 $\mu \mathrm{L}$ solution of Au NPs coated with phenylalanine. As shown in Fig. $3 \mathrm{~d}$, in the absence of the drug the fluorescence intensity of the N-GQDs is decreased. This observation can be explained based on the Forster resonance energy transfer mechanism that the excited electrons in N-GQDs are adsorbed by the Au NPs coated with phenylalanine, and the resulted cavity in the ground state is replaced with the resonance electron of the compound. The result of these electron transitions and resonance is selfabsorption and fluorescence quenching. The addition of piroxicam to this mixture causes the release of the Au NPs from the N-GQDs surface, followed by the retrieve of the fluorescence intensity. The release of gold nanoparticles from the N-GQDs surface can be due to the formation of the hydrogen bonds between phenylalanine and the drug. This experiment confirms the important role of the Au NPs coated with phenylalanine in the designed method for the determination of piroxicam. The possible mechanism of interaction of N-GQDs with Au NPs coated with phenylalanine and piroxicam is shown in Fig. 4.

\section{Optimization of experimental conditions}

The influence of $\mathrm{pH}$ on the analytical signal was investigated by changing the $\mathrm{pH}$ within the range of 4.09.0 using the phosphate buffer. As shown in Fig. $5 \mathrm{a}$, the analytical signal is maximized at the $\mathrm{pH}$ of 7.0 that was chosen as the optimal $\mathrm{pH}$ for further studies. Piroxicam is a diprotic amphoteric drug with $\mathrm{pKa}_{\mathrm{s}}$ values of 1.86 and 5.46 . So, when the $\mathrm{pH}$ of a solution is greater than its $\mathrm{pKa}_{2}$, the anionic form of piroxicam predominate in the solution. Thus, at the $\mathrm{pH}$ of 7.0, the drug is mainly in its anionic form and the electron transfer takes place from the piroxicam to the electropositive Au NPs containing empty $d$ orbital and the analytical signal is maximized. However, at higher $\mathrm{pH}$, the electron transfer to the empty $\mathrm{d}$ orbital is completed resulting in the decrease in analytical signal.

The effect of N-GQDs volume in the range of 100.0-700.0 $\mu \mathrm{L}$ on the analytical signal was considered. It was observed that (Fig. 5b) in the presence of a fixed amount of piroxicam, the analytical signal increases by increasing the volume of N-GQDs up to $500 \mu \mathrm{L}$ and then decreases. The decrease in the signal at a higher N-GQDs volume can be due to the increase in the ratio of N-GQDs to the fixed concentration of piroxicam and Au NPs coated with phenylalanine. Thus, the volume of $500.0 \mu \mathrm{L}$ was used as the optimal volume of $\mathrm{N}-\mathrm{GQDS}$.

The influence of the Au NPs coated with phenylalanine volume in the range of 100.0-600.0 $\mu \mathrm{L}$ on the analytical signal was examined. The results of this study (Fig. 5c) indicate that the analytical signal increases up to the $300.0 \mu \mathrm{L}$ of nanoparticles and then decreases. The decrease in analytical signal in a volume greater than $300.0 \mu \mathrm{L}$ can be attributed to the increase of volume of Au NPs coated with 
phenylalanine in comparison to the fixed concentration of the drug, in which case the extra gold nanoparticles adsorb to the surface of N-GQDs and reduce the analytical signal. Therefore, a volume of $300.0 \mu \mathrm{L}$ was selected as the optimal volume.

The contact time between the drug and N-GQDs in the presence of Au NPs coated with phenylalanine was also optimized by varying the contact time within the range of 2-25 minutes. An increase in contact time up to 10 minutes, increased the analytical signal and then remained constant. In other words, for complete interaction of drug with phenylalanine and release of the Au NPs from the surface of N-GQDs, a contact time of 10.0 minutes is required.

\section{Selectivity studies}

To evaluate the proposed method selectivity for measuring piroxicam, the influence of some drugs in the same category with piroxicam such as naproxen, aspirin, and ibuprofen, as well as other drugs (lamotrigine, carbamazepine, tetracycline, and diclofenac) and compounds (glycine, ascorbic acid, histidine, uric acid, and glucose) and metal ions including $\mathrm{Ca}^{2+}, \mathrm{Na}^{+}$, and $\mathrm{K}^{+}$on the analytical signal was examined. An error of less than $5 \%$ was considered at the random experimental error. For this purpose, the standard solution of each of these materials with a concentration of $110.0 \mathrm{nmol} \mathrm{L}^{-1}$ was measured separately by the designed method. Based on the results of this study (Fig. 3S), the effect of most of these species with a concentration of 10 times of the piroxicam on the analytical signal is negligible and the analytical signal of aspirin, ibuprofen, and naproxen (at a concentration of $110.0 \mathrm{nmol} \mathrm{L}^{-1}$ ) is about half of the piroxicam analytical signal with the concentration of $11.0 \mathrm{nmol} \mathrm{L^{-1 }}$. Thus, the designed method has good selectivity for the measurement of piroxicam.

\section{Analytical features}

At the optimized conditions, the analytical performance of the developed method for the determination of piroxicam was investigated. The signal was linearly increased with increasing the piroxicam concentration within the range of 2.0 to $35.0 \mathrm{nmol} \mathrm{L}^{-1}$ (Fig. 6) with the equation of $y=0.0048 x+0.0169$ and coefficient of determination of 0.9949 (where $y$ is the analytical signal $\left(F-F_{0}\right) / F_{0}$ and $x$ is the piroxicam concentration $\left(\mathrm{nmol} \mathrm{L}^{-1}\right)$. The detection and quantification limits were found to be 0.11 and $0.36 \mathrm{nmol} \mathrm{L}^{-1}$, respectively. The intra and inter-day precisions were determined by six independent replicate measurements of piroxicam at $11.0 \mathrm{nmol} \mathrm{L}^{-1}$ and were 2.2 and $4.5 \%$, respectively.

\section{Real sample analysis}

The capability of the developed method for measurement of the piroxicam in plasma and urine samples was evaluated. For investigation of the reliability of the method, each sample was spiked at two concentration levels of piroxicam and the recoveries were of the added analyte was calculated. The results (Table 1) exhibited that the recoveries of all spiked samples are in the range of $94.5-101.8 \%$, indicating the accuracy of the developed method for the quantitative piroxicam measurements in these 
samples types. The reliability of the method was also confirmed by analyzing the commercial capsules of piroxicam. The mean amount of piroxicam was determined to be $9.7 \pm 0.2 \mathrm{mg} / \mathrm{capsule}$ which at $95 \%$ confidence level is in good agreement with the value claimed $(10.0 \mathrm{mg} / \mathrm{tablet})$.

Table 1

Determination of piroxicam in real samples

\begin{tabular}{|llll|}
\hline Sample & Added $\left(\mathrm{nmol} \mathrm{L}^{-1}\right)$ & Found* $\left(\mathrm{nmol} \mathrm{L}^{-1}\right)$ & Recovery (\%) \\
\hline Plasma & - & N.D & - \\
\hline & 9.0 & $8.6 \pm 0.3$ & 95.6 \\
\hline Urine & - & $11.2 \pm 0.5$ & 101.8 \\
\hline & 9.0 & N.D & - \\
\hline & 11.0 & $8.8 \pm 0.4$ & 97.8 \\
\hline * Mean and standard deviation of three independent analysis. \\
\hline
\end{tabular}

\section{Comparison of the analytical performance of the method with other reported methods}

The analytical performance of the developed method was compared with some other reported methods for the measurement of piroxicam. The results of this study (Table 2) indicate that the limit of detection of the designed method is lower than most of the compared methods and has a reproducibility better or comparable with them. In addition, the determination of piroxicam with this designed optical sensor is simple and fast. 
Table 2

Comparison of the developed method with other reported methods for determination of piroxicam

\begin{tabular}{|c|c|c|c|c|c|}
\hline Method & $\begin{array}{l}\text { Linear range } \\
(\mathrm{nmol} \mathrm{L}-1)\end{array}$ & $\begin{array}{l}\text { Detection limit } \\
\left(\mathrm{nmol} \mathrm{L}{ }^{-1}\right)\end{array}$ & $\begin{array}{l}\text { RSD } \\
(\%)\end{array}$ & Real sample & Reference \\
\hline LC & $3.0-18000$ & 0.011 & - & Serum & [27] \\
\hline CV & $0.5-100.0$ & 0.11 & 3.11 & Serum & [28] \\
\hline CV-SWV & $8.7 \times 10^{2}-2.6 \times 10^{4}$ & $1 \times 10^{2}$ & 3.8 & River water & [29] \\
\hline PV & $0.5 \times 10^{3}-5.0 \times 10^{4}$ & 50.0 & 2.22 & Serum & [30] \\
\hline DPV & $100.0-7.0 \times 10^{4}$ & 40.0 & 4.2 & Serum & [31] \\
\hline PL & $3.01 \times 10^{3}-6.04 \times 10^{4}$ & $1.47 \times 10^{3}$ & 4.1 & Drug & [32] \\
\hline LC-MS-MS & $11.0-56.0$ & 2.0 & 3.0 & Serum & [33] \\
\hline SFC-MS-MS & $11.0-11.25 \times 10^{3}$ & - & 6.6 & Serum & [34] \\
\hline PL & $2.0-35.0$ & 0.11 & 2.2 & Urine-Serum & Present study \\
\hline
\end{tabular}

\section{Conclusion}

In this study, N-GQDs were synthesized and an optical sensor was designed for the determination of piroxicam based on the off-on fluorescence intensity of N-GQDs using Au NPs coated with phenylalanine. The developed method was successfully applied to the measurement of piroxicam in the complex matrices of human urine and serum samples. The structural properties of N-GQDs were investigated by various spectroscopic methods. The developed method possesses the advantages of proper detection limit without the need for separation and preconcentration step, good selectivity, and simplicity.

\section{Declarations}

\section{Funding}

The authors declare that no funds, grants, or other support were received during the preparation of this manuscript

\section{Competing Interests}


The authors declare that is no financial and personal relationships with other people or organizations that can inappropriately influence our work, there is no professional or other personal interest of any nature or kind in any product, service and/or company.

\section{Author Contributions}

All authors contributed to the study conception and design. Material preparation, data collection and analysis were performed by Maryam Moallemi Bahmani, Ali Mohammad Haji Shabani*, Shayessteh Dadfarnia* and Roya Afsharipour. The first draft of the manuscript was written by Roya Afsharipour and all authors commented on previous versions of the manuscript. All authors read and approved the final manuscript."

\section{Data Availability}

The datasets generated during and/or analysed during the current study are not publicly available but are available from the corresponding author on reasonable request."

\section{Ethics approval}

This is an observational study. The XYZ Research Ethics Committee has confirmed that no ethical approval is required.

\section{Consent to participate \& Consent to publish}

This study was not involved human.

\section{References}

1. Tabrizi AB (2007) A simple spectrofluorimetric method for determination of piroxicam and propranolol in pharmaceutical preparations. J Food Drug Anal 15:242-248

2. Damiani PC, Bearzotti M, Cabezón M, Olivieri AC (1998) Spectrofluorometric determination of piroxicam. J Pharm Biomed Anal 17:233-236

3. Sherman KE, Jones C (1992) Hepatotoxicity associated with piroxicam use. Gastroenterology 103:354-355

4. Kormosh ZA, Hunka IP, Bazel YR (2011) Spectrophotometric determination of piroxicam. J Anal Chem 66:378-383

5. B. Boneschans, A. Wessels, J.V. Staden, M. Zovko, B. Zorc, J. Bergh (2003), Piroxicam benzoate synthesis, HPLC determination and hydrolysis, Drug Dev. Ind. Pharm. 29 (2003) 155-160.

6. Escandar GM (1999) Spectrofluorimetric determination of piroxicam in the presence and absence of $\beta$-cyclodextrin. Analyst 124:587-591

7. Dal AG, Oktayer Z, Doğrukol-Ak D (2014) Validated method for the determination of piroxicam by capillary zone electrophoresis and its application to tablets. J Anal Methods Chem 2017:352698 
8. Wong A, Santos AM, Fatibello-Filho O (2017) Determination of piroxicam and nimesulide using an electrochemical sensor based on reduced graphene oxide and PEDOT:PSS. J Electroanal Chem 799:547-555

9. Shahrokhian S, Jokar E, Ghalkhani M (2010) Electrochemical determination of piroxicam on the surface of pyrolytic graphite electrode modified with a film of carbon nanoparticle-chitosan. Microchim Acta 170:141-146

10. Liu J, Xin XY, Zhou H, Zhang SS (2015) A ternary composite based on graphene, hemin, and gold nanorods with high catalytic activity for the detection of cell surface glycan expression. Chem Eur $J$ 21:1908-1914

11. Li XR, Kong FY, Liu J, Liang TM, Xu JJ, Chen HY (2012) Synthesis of potassium modified graphene and its application in nitrite-selective sensing. Adv Funct Mater 22:1981-1988

12. Hao N, Zhang X, Zhou Z, Qian J, Liu Q, Chen SB, Zhang Z, Wang K (2017) Threedimensional nitrogendoped graphene porous hydrogel fabricated biosensing platform with enhanced photoelectrochemical performance. Sens Actuators B Chem 250:476-483

13. Sadanandhan NK, Cheriyathuchenaaramvalli M, Devaki SJ, Menon ARR (2017) PEDOT-reduced graphene oxide-silver hybrid nanocomposite modified transducer for the detection of serotonin, $\mathrm{J}$ Electroanal Chem 794:244-253

14. S. Fathalipour, E. Abdi, Glycine-assisted aqueous suspension of reduced graphene oxide/Ag nanocomposite via in situ reduction at room temperature: synthesis and electroactivity behavior, Synth. Met. 221 (2016) 159-168.

15. Khan A, Khan AAP, Asiri AM, Abu-Zied BM (2016) Green synthesis of thermally stable ag-rGO-CNT nanocomposite with high sensing activity. Compos Part B-Eng 86:27-35.

16. Zhang H, Zhang LL, Han YJ, Yu Y, Xu M, Zhang XP, Huang L, Dong SJ (2017) RGO/ Au NPs/N-doped CNTs supported on nickel foam as an anode for enzymatic biofuel cells. Biosens Bioelectron 97:3440

17. Jiang X, Kou Y, Lu J, Xue Y, Wang M, Tian B, Tan L (2020) Fluorescence "on-off-on" Assay of Copper Ions and EDTA Using Amino-Functionalized Graphene Quantum Dots. J Fluoresc 30:301-308.

18. Kaviani R, Ghaffary S, Jouyban A, Shayanfar A (2020) Developing an Analytical Method Based on Graphene Quantum Dots for Quantification of Deferiprone in Plasma. J Fluoresc 30:591-600.

19. Ma CB, Zhu ZT, Wang HX, Huang X, Zhang X, Qi XY, Zhang HL, Zhu YH, Deng X, Peng Y, Han Y, Zhang $H$ (2015) A general solid-state synthesis of chemically-doped fluorescent graphene quantum dots for bioimaging and optoelectronic applications. Nanoscale 7:10162-10169

20. Sun HJ, Zhao AD, Gao N, Li K, Ren JS, Qu XG (2015) Deciphering a nanocarbon-based artificial peroxidase: chemical identification of the catalytically active and substrate-binding sites on graphene Quantum dots. Angew Chem Int Ed 54:7176-7180

21. Afsharipour R, Shabani AM, Dadfarnia S, Kazemi E (2020) Selective fluorometric determination of sulfadiazine based on the growth of silver nanoparticles on graphene quantum dots. Microchim Acta $2020187: 1-8$ 
22. Afsharipour R, Dadfarnia S, Shabani AM, Kazemi E, Pedrini A, Verucchi R (2021) Fabrication of a sensitive colorimetric nanosensor for determination of cysteine in human serum and urine samples based on magnetic-sulfur, nitrogen graphene quantum dots as a selective platform and $\mathrm{Au}$ nanoparticles. Talanta 226:122055

23. He YZ, Sun J, Feng DX, Chen HQ, Gao F, Wang L (2015) Graphene Quantum dots: highly active bifunctional nanoprobes for nonenzymatic photoluminescence detection of hydroquinone. Biosens Bioelectron 74:418-422

24. Yuan FL, Ding L, Li YC, Li XH, Fan LZ, Zhou SX, Fang DC, Yang SH (2015) Multicolor fluorescent graphene quantum dots colorimetrically responsive to all-pH and a wide temperature range. Nanoscale 7:11727-11733

25. Duan L, Du X, Zhao H, Sun Y, Liu W (2020) Sensitive and selective sensing system of metallothioneins based on carbon quantum dots and gold nanoparticles. Anal Chim Acta 1125:177186

26. Wang X, Guo XQ (2009) Ultrasensitive $\mathrm{Pb}^{2+}$ detection based on fluorescence resonance energy transfer (FRET) between quantum dots and gold nanoparticles. Analyst 134:1348-1354

27. Sultana N, Arayne MS, Ali SN (2013) Liquid chromatographic method for the simultaneous determination of captopril, piroxicam, and amlodipine in bulk drug, pharmaceutical formulation, and human serum by programming the detector. J Sep Sci 36:3176-3183

28. Gholivand MB, Malekzadeh G, Derakhshan AA (2014) Boehmite nanoparticle modified carbon paste electrode for determination of piroxicam. Sens Actuators B Chem 201:378-386

29. Wong A, Santos AM, Fatibello-Filho O (2017) Determination of piroxicam and nimesulide using an electrochemical sensor based on reduced graphene oxide and PEDOT: PSS. J Electroanal Chem 799:547-555

30. Shaikh T, Talpur FN, Khaskeli AR, Agheem MH, Shah MR, Sherazi TH, Siddiqui S (2017) Ultrasensitive determination of piroxicam at diflunisal-derived gold nanoparticle-modified glassy carbon electrode. J Electron Mater 46:5957-5966

31. Babaei A, Afrasiabi M (2015) A glassy carbon electrode modified with MCM-41/nickel hydroxide nanoparticle/multiwalled carbon nanotube composite as a sensor for the simultaneous determination of dopamine, piroxicam, and cefixime. Ionics 21:1731-1740

32. Kormosh ZA, Hunka IP, Bazel YR (2011) Spectrophotometric determination of piroxicam. J Anal Chem 66:378-383

33. Shirako J, Kawasaki M, Komine K, Kunisue Y, Terada M, Sasaki C, Irie W, Murakami C, Tonooka K, Tomobe K, Shinozuka T (2013) Simultaneous determination for oxicam non-steroidal antiinflammatory drugs in human serum by liquid chromatography-tandem mass spectrometry. Forensic Sci Int 227:100-102

34. Li X, Gao Y, Liu J, Zhang G, Zhang T (2018) A rapid analysis of piroxicam in beagle plasma applying evaporation-free liquid-liquid extraction by supercritical fluid chromatography-tandem mass spectrometry. J Chromatogr B 1100:93-99

Page $13 / 17$ 
Figures
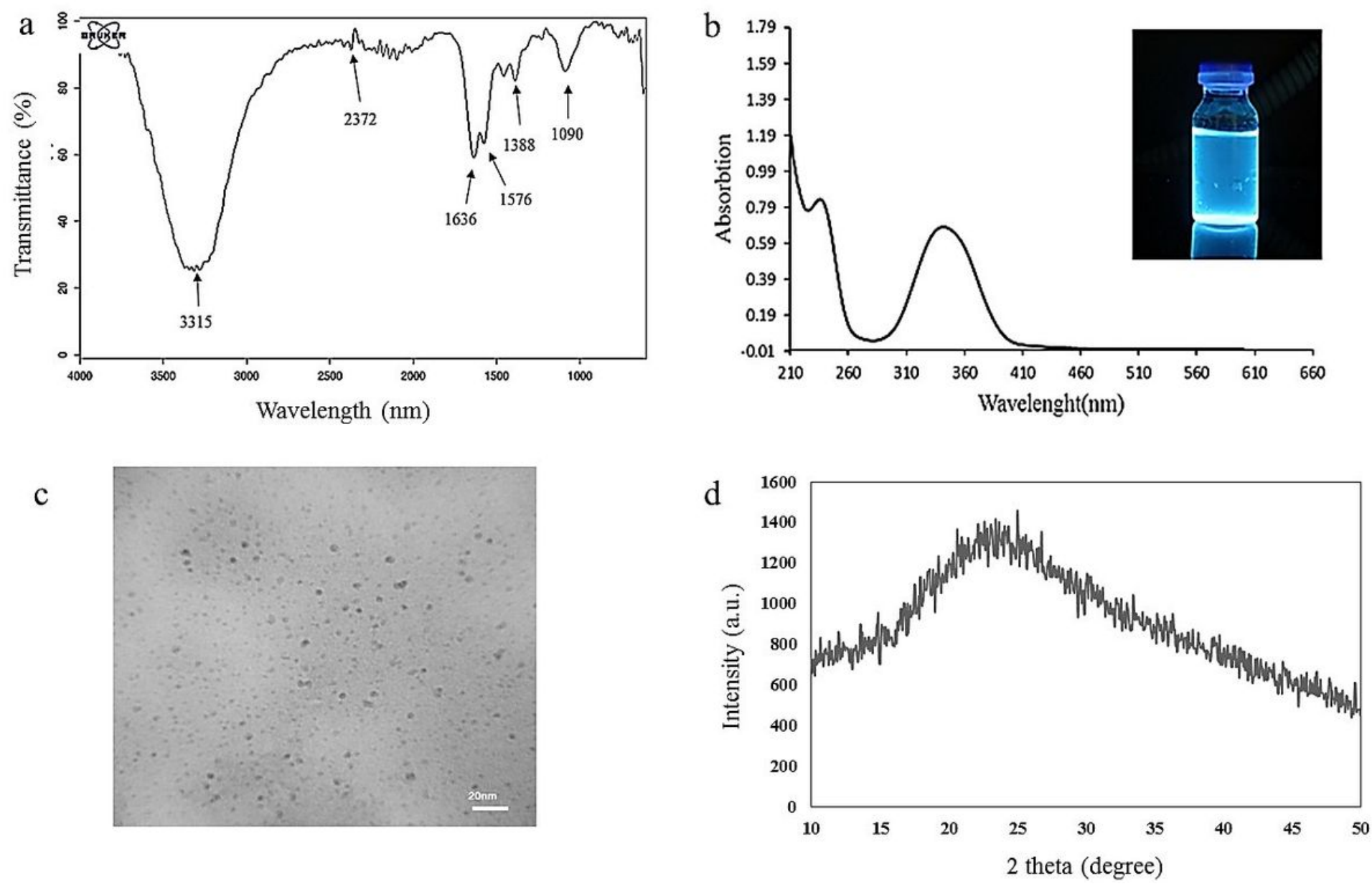

Figure 1

(a) FT-IR spectrum of N-GQDs, (b) UV-Visible spectrum of N-GQDs, (c) TEM image of N-GQDs, (d) XRD pattern of N-GQDs<smiles>O=CC(C(=O)O)C(c1ccccc1)c1ccccc1</smiles>

Phenylalanine
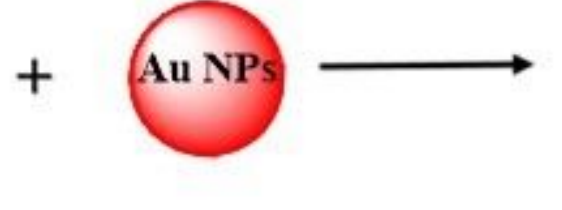

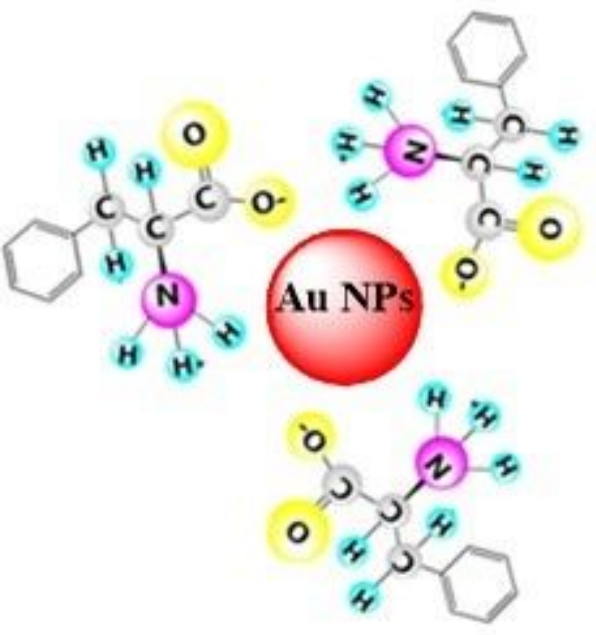

Figure 2 
Schematic illustration of forming Au NPs coated with phenylalanine

a)

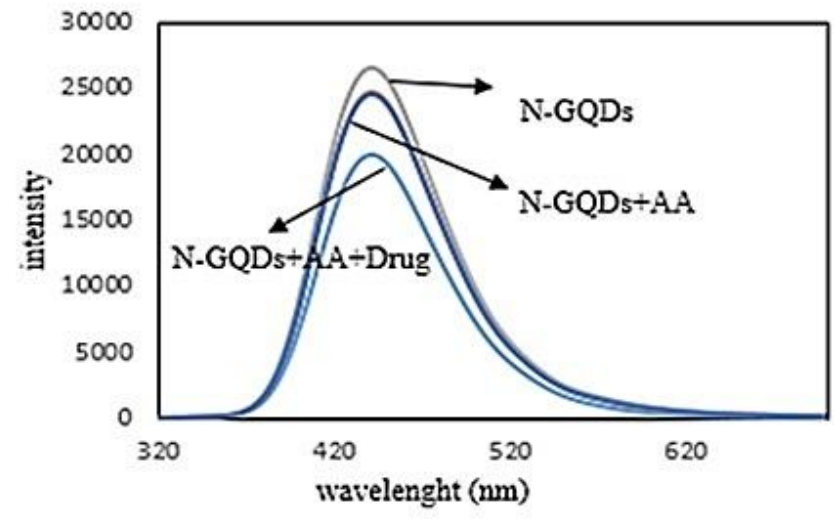

c)

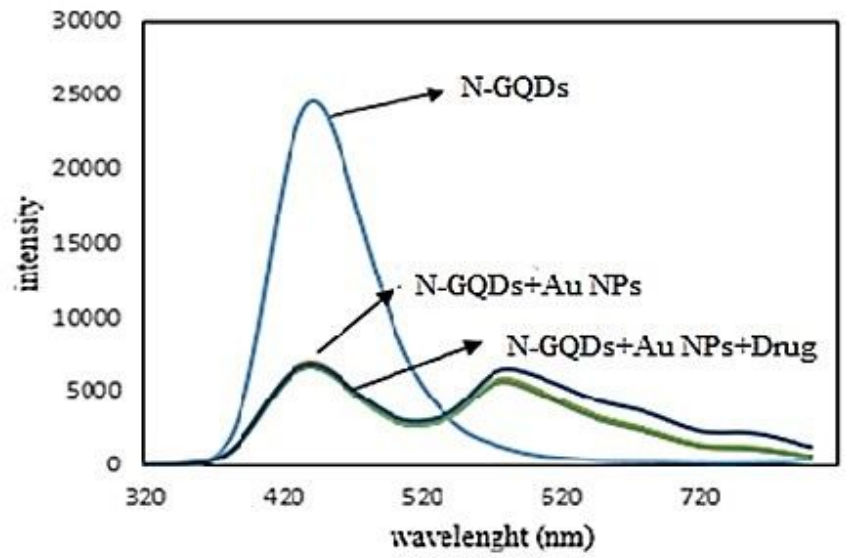

b)

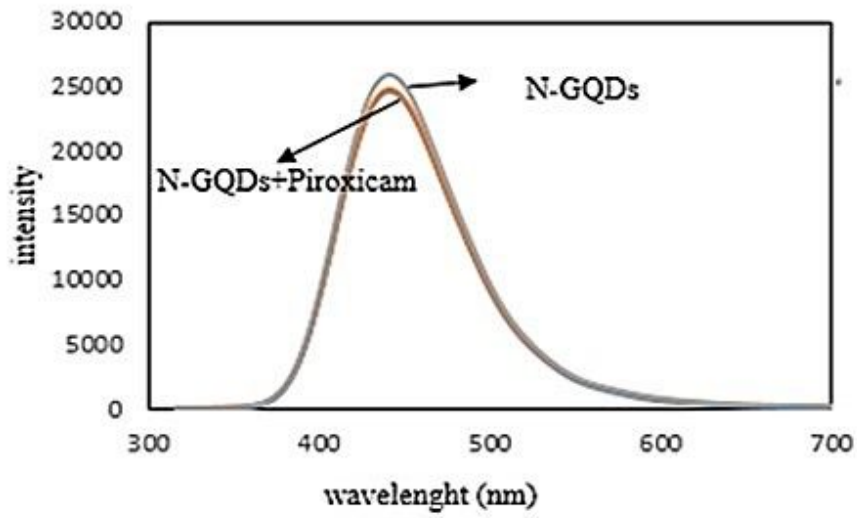

d)

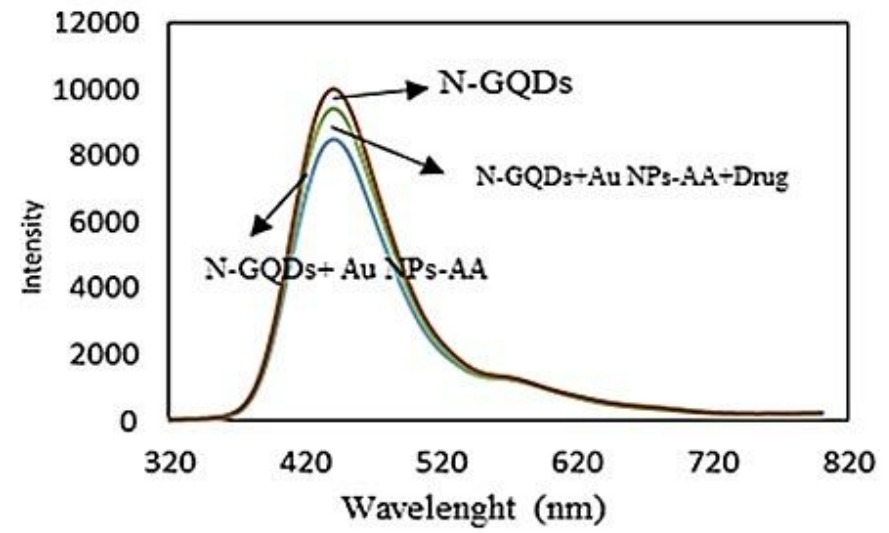

Figure 3

(a) Fluorescence spectra of N-GQDs after adding phenylalanine and phenylalanine and piroxicam, (b) Fluorescence spectra of N-GQDs after adding piroxicam, (c) Fluorescence spectra of N-GQDs after adding Au NPs and Au NPs and piroxicam (d) Fluorescence spectra of N-GQDs after adding Au NPs coated with phenylalanine and Au NPs coated with phenylalanine and piroxicam

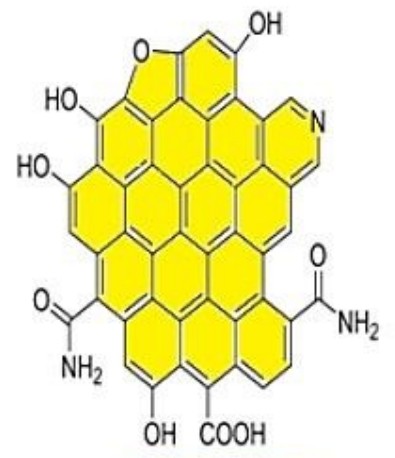

Strong emission

22 Phenylalanine

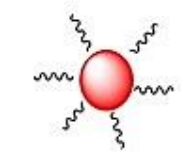

Phenylalanine capped Au NPs

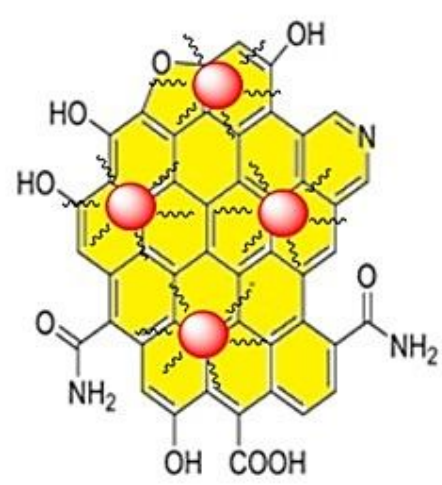

\section{Fluorescence off}

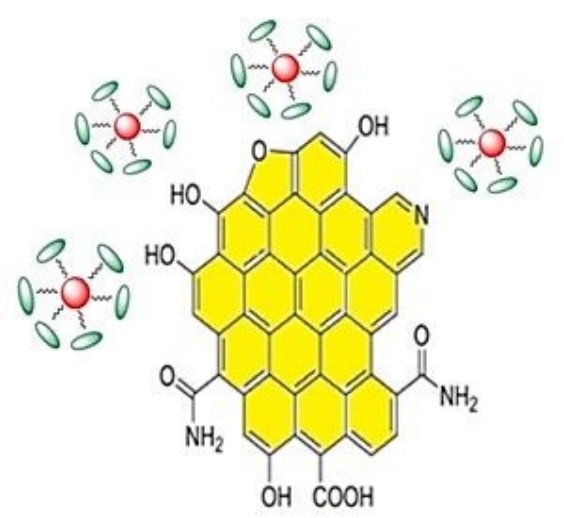

Fluorescence on

Piroxicam 
Figure 4

Schematic representation of piroxicam determination using Au NPs coated with phenylalanine and NGQDs as a fluorescent nanoprobe
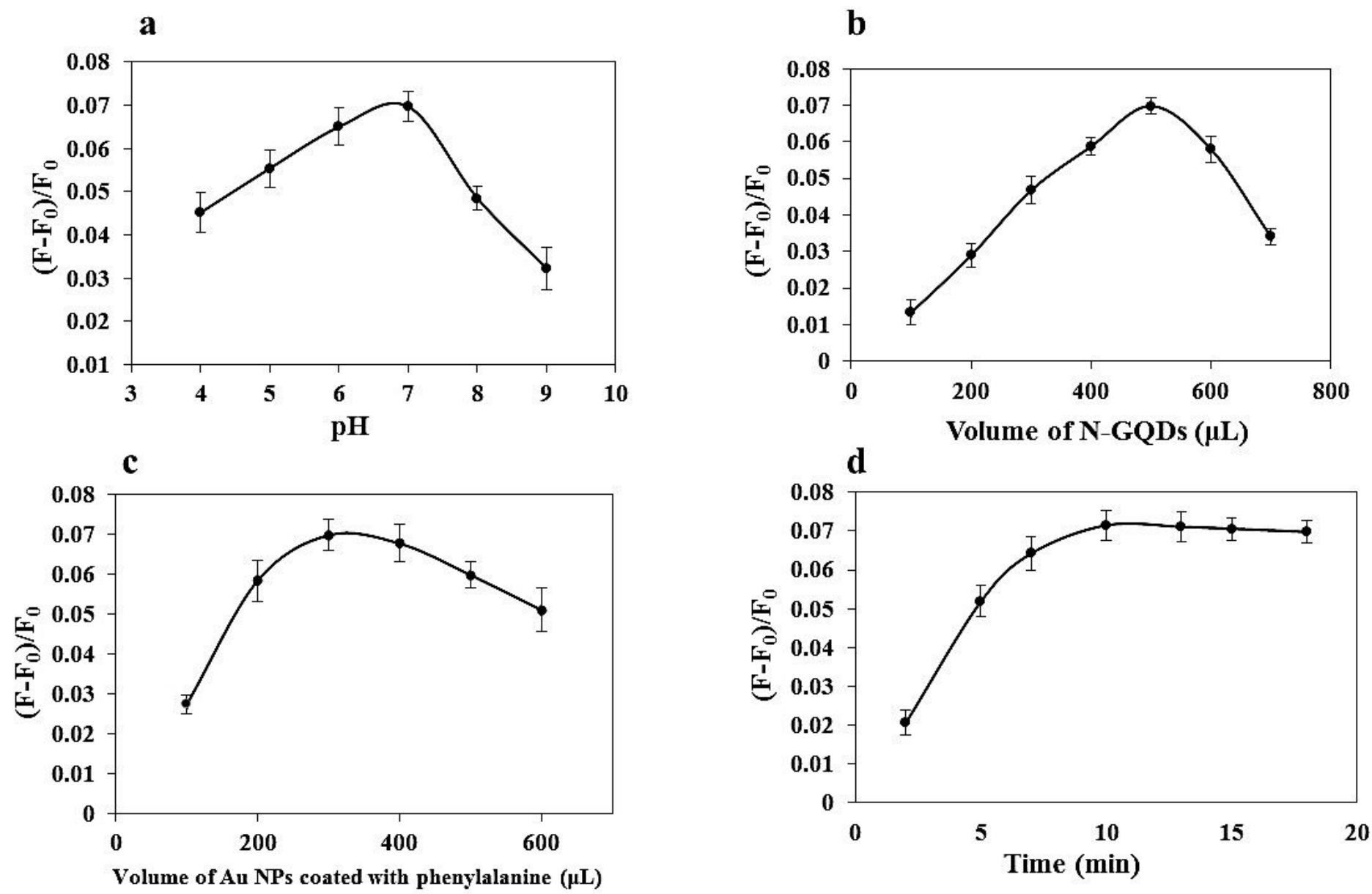

\section{Figure 5}

(a) Effect of pH value. Conditions: NGQDs (500.0 $\mu \mathrm{L})$, Au NPs coated with phenylalanine $(500.0 \mu \mathrm{L})$, piroxicam (11.0 nmol L-1), time (15.0 min), (b) Effect of NGQDs volume. Conditions: $\mathrm{pH}=7.0$ and other conditions as stated in (a), (c) Effect of Au NPs-coated with phenylalanine volume. Conditions: N-GQDs $(500.0 \mu \mathrm{L})$ and other conditions as stated in (b), (d) Effect of time. Conditions: Au NPs coated with phenylalanine $(300 \mu \mathrm{L})$ and other condition as stated in (c) 

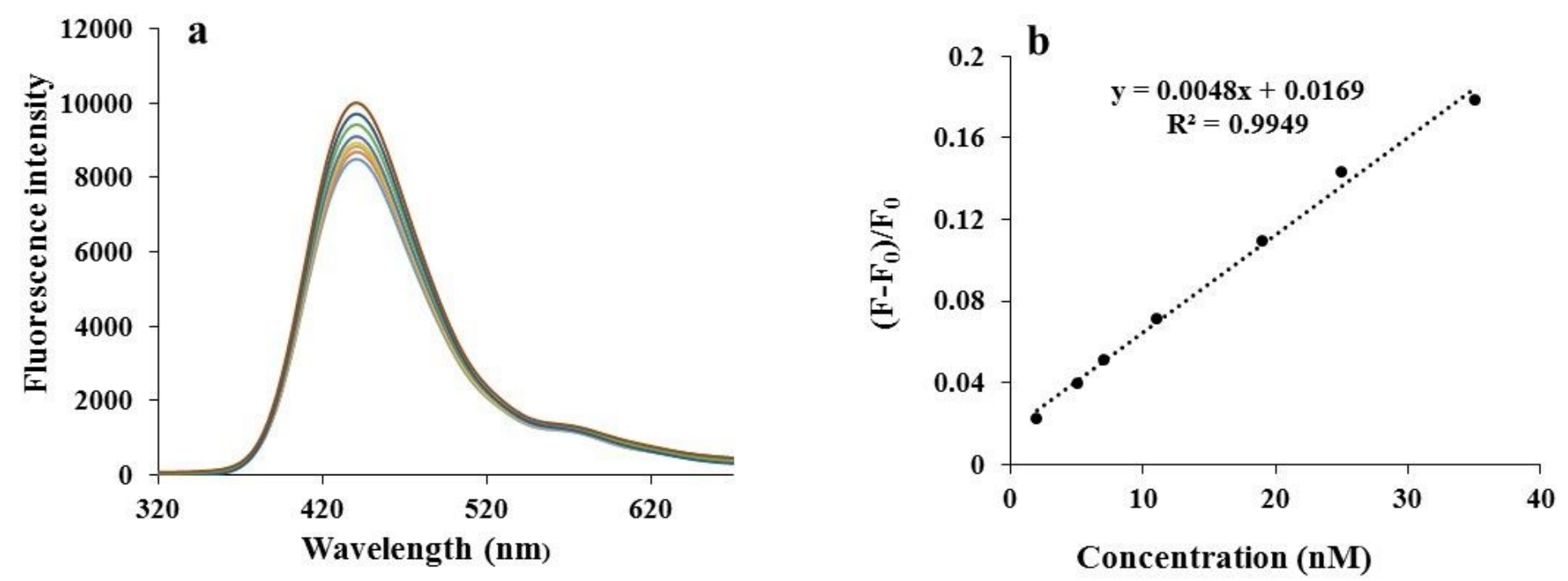

Figure 6

(a) Fluorescence spectra of the developed method at various concentrations of piroxicam, (b) Calibration plot of the analytical signal

\section{Supplementary Files}

This is a list of supplementary files associated with this preprint. Click to download.

- suplementry.docx 